\section{The clinical fellows project: emergence of the FY3 year?}

Since August 2017, Sheffield Health and Social Care have begun providing a new non-training post in psychiatry. This was done with multiple aims, including attempting to increase the uptake of core psychiatry training jobs in South Yorkshire by giving a positive experience of working in a psychiatric setting and improving local clinical care with an increase in the number of doctors working at SHO/CT1 level. The non-training posts were described as clinical fellowships and were open to doctors who had not yet undertaken higher training in psychiatry.

We, Drs Raha and Heminway, were among the first people to take up these clinical fellowship posts and were therefore in a prime position to review how the project is being received and some of its outcomes. Since August 2017, 15 clinical fellows have been recruited, largely in the 'FY3 gap' but with some coming from general practitioner (GP) training. Dr Raha entered after having been a GP trainee and Dr Heminway from a postfoundation training break.

The clinical fellowship consists of a non-training junior doctor post, working at FY2/CT1-equivalent level in psychiatry. A number of rotational placements were created, including those based in general adult, older adult and addiction psychiatry, across both community and in-patient settings. A key benefit that was felt by the clinical fellows was that there were additional training opportunities given - a split of $80 \%$ clinical to $20 \%$ non-clinical time allowance, access to weekly continuing professional development (CPD) sessions, weekly supervision with a consultant psychiatrist and attending the core psychiatry training course for MRCPsych examinations. Clinical fellows have also since set up their own peer group to improve CPD and training opportunities for future clinical fellows.

The experience has been invaluable, not just for ourselves but also for other clinical fellows involved in the project; this has been borne out by the increasing uptake of core psychiatry training posts by the clinical fellows. Of the 2017-2018 cohort, half entered core psychiatry training, one entered GP training and one left owing to long-term sickness. Half the 2018-2019 cohort were also considering entering psychiatric training at the end of the clinical fellowship posting.

The culmination of this experience was the opportunity to present this project at the Choose Psychiatry meeting of the Royal College of Psychiatrists earlier this year. It was well received, owing to the fact that it was both improving access to psychiatry and improving junior doctors' opinion of psychiatric training and work (including those leaving the post to take up GP jobs). It was also highlighted that many junior doctors are no longer entering core training directly after foundation training. This clinical fellowship has therefore been an excellent way to accommodate this trend and provide a springboard for increasing access to and awareness of psychiatric training.

Anirban Raha, CT2, Sheffield Health and Social Care NHS Foundation Trust, email: anirban.raha@shsc.nhs.uk; Robert Heminway, CT1, Sheffield Health and Social Care NHS Foundation Trust, email: robert.heminway@shsc nhs.uk

doi:10.1192/bjb.2020.16 (c) The Authors 2020. This is an Open Access article distributed under the terms of the Creative Commons Attribution licence (http://creativecommons.org/ licenses/by/4.0/), which permits unrestricted re-use, distribution, and reproduction in any medium, provided the original work is properly cited.

\section{A brief history of the evolution of the Parliamentary Scholar Scheme}

We were really pleased to read about the Parliamentary Scholar Scheme and how successful it has become - thank you for your thorough overview of this invaluable opportunity for speciality trainees in psychiatry. ${ }^{1}$ The scheme can trace its origins back to a pilot scheme set up by Baroness Hollins in 2012, when KJ was one of three trainees at higher speciality trainee level in Psychiatry of Intellectual Disability or Child and Adolescent Psychiatry. He joined her team at the House of Lords one day a week for four months as a special interest session in Parliamentary affairs.

Similar to the current scheme, the role was to assist in the research and preparation of Parliamentary business, including speeches and written questions delivered by Baroness Hollins in the House of Lords. A wide range of new skills were developed by trainees, including understanding Parliamentary processes; developing communication skills beyond the usual boundaries of medicine; and making alliances with individuals and organisations from different professional and lay backgrounds in order to accomplish shared goals for the benefit of the vulnerable groups we support.

The work Baroness Hollins and her team did to represent the voice of people with an intellectual disability and/or mental illness at a Parliamentary level was truly inspiring, and the opportunity to be part of this team, advocating for our patient groups at a national level, was extremely rewarding and a great privilege. As you mentioned in your article, the opportunity opened our eyes to the substantial influence that medically trained professionals can have in contributing to political and managerial discourse and decision-making, in the widest sense.

$A B$, who was the Speciality Advisory Committee Chair, Intellectual Disability Faculty, Royal College of Psychiatrists, at the time, believes that this unparalleled and unique training opportunity and experience had a long-lasting effect on the careers and overall personal development of trainees who were successful in the selection process and then went on to train, refine their skills, and learn about Parliamentary processes and how to influence policy-making, supervised by Baroness Hollins.

$\mathrm{KJ}$ feels it has influenced his subsequent decisions to pursue dual training in Intellectual Disability (after a first CCT in Child and Adolescent Psychiatry), act as trainee representative in the Royal College Faculty Executive and more recently join the Royal College of Psychiatrists Leadership and Management Scheme. Another colleague from this early form of the scheme went on to join the Royal College of Psychiatrists Westminster Parliamentary Committee.

We would like to take this opportunity to sincerely thank Baroness Hollins for setting up this scheme and encourage all trainees with an interest in this area to consider the scheme as a unique and invaluable opportunity. We believe this pioneering scheme in all its iterations has already left an important legacy within our generation of psychiatrists and beyond.

Keir Jones, ST4 in Psychiatry of Intellectual Disability (formerly Consultant Child and Adolescent Psychiatrist), Leicestershire Partnership NHS Trust, 
email: keir.jones@leicspart.nhs.uk; Asit Biswas, Vice-Chair of Psychiatry of Intellectual Disability Faculty Executive, Royal College of Psychiatrists, Consultant Psychiatrist of Intellectual Disability, Leicestershire Partnership NHS Trust, and Honorary Associate Professor, University of Leicester.

1 Perry J, Lomax P, Taylor F, Howson S, McCurdy K. The Parliamentary Scholar Scheme: a way to engage doctors in healthcare policy and politics. BJPsych Bull 2019; doi: 10.1192/bjb.2019.76.

\section{doi:10.1192/bjb.2020.17}

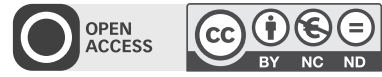

(c) The Authors 2020. This is an Open Access article, distributed under the terms of the Creative Commons Attribution-NonCommercial-NoDerivatives licence (http://creativecommons.org/licenses/by-nc-nd/4.0/), which permits noncommercial re-use, distribution, and reproduction in any medium, provided the original work is unaltered and is properly cited. The written permission of Cambridge University Press must be obtained for commercial re-use or in order to create a derivative work.

\section{Obituary}

\section{Hanafy Ahmed Youssef, DM, MRCS, FRCPsych}

\author{
Formerly Consultant Psychiatrist, St Davnet's Hospital, \\ Monaghan, Ireland
}

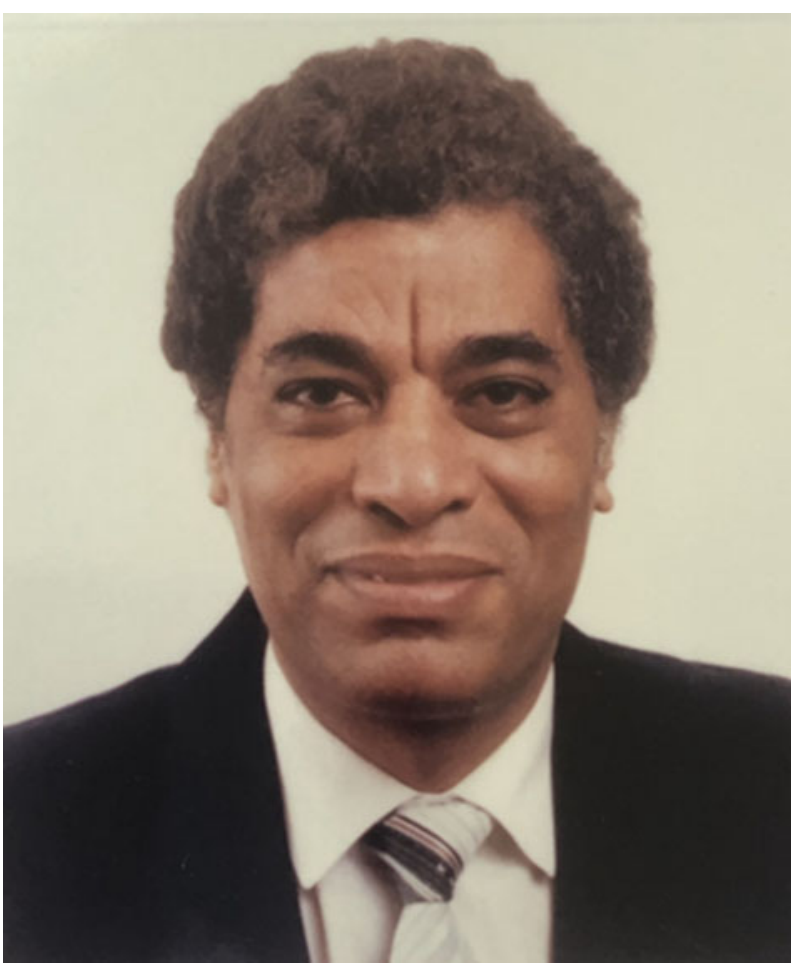

Hanafy Youssef, who died at the age of 80 on 21 January 2019 was a leading figure in Irish psychiatry. With his colleague John Owens, he set up the first community-based psychiatric service in Ireland. He was able to show that this pattern of service led to better outcomes for patients and their families. As a result, a number of other community-based services in Ireland and the UK were established.

Over a 20-year period in the 1980s and 1990s, he developed a highly productive research relationship with Professor John Waddington of the Royal College of Surgeons, Ireland. Together they published many papers on aspects of psychotic illness occurring in patients referred to the Cavan/Monaghan psychiatric services. Hanafy Youssef contributed to over 60 publications in the fields of psychotic illness, neurodevelopmental psychiatry and psychopharmacology. The relationship between the Cavan/Monaghan psychiatric services and the Department of Psychopharmacology at the Royal College of Surgeons, Ireland, continues to this day.

Hanafy had a strong interest in postgraduate psychiatric education. He was clinical tutor at St Davnet's Hospital and a member of the Irish Psychiatric Training Committee. He had a great capacity for explaining difficult concepts to medical and nursing staff. Indeed, as an educator, he was at the forefront of psychiatric education in Ireland. He was also a mentor to many overseas doctors who worked with him. He was an inspiration to them as they saw him become a leader in his specialty. He was a particularly strong supporter of women in medicine at a time when women doctors were still finding it difficult to climb the career ladder.

He had a great interest in the development of psychiatric services in less economically developed countries. In the 1970s he spent time in Zambia, developing clinics in rural areas and helping to confront the stigma often attached to psychiatric illnesses. From 1994, he spent 2 years in a professorial post in Trinidad and Tobago, where he helped improve the quality and prominence of undergraduate psychiatry training. He felt this was crucial if the finest doctors were to be recruited into the specialty. He then returned to take up a consultant post in liaison and general psychiatry at Addenbrooke's, a teaching hospital within Cambridge University Hospitals NHS Foundation Trust. In addition, he carried out voluntary work with charities in Yemen and Libya, providing both general medical and psychiatric care in areas where there was a shortage of doctors.

Hanafy was also a philanthropist, fiercely passionate about social justice, who took seriously the welfare of the less privileged. Together with his siblings he established Latifa's Orphanage House and a mosque in the suburbs of Alexandria. He worked tirelessly for the underprivileged and would offer free medical care to any in need.

Whether they spoke to him in person, heard him speak at international conferences or were his patients, Hanafy Youssef left a lasting impression on all he met. His knowledge, dedication to his field and commitment to his patients were widely appreciated.

He came from humble beginnings. He was born on 14 January 1939 in Alexandria, Egypt, to Ahmed Mahmoud and Latifa (née Taher). His father ran a hunting and tackle store there. Hanafy studied medicine at Alexandria University and undertook postgraduate training in the Department of Psychiatry at the University of Cairo. At university, he led an extremely active life, both in cultural pursuits and in sport. $\mathrm{He}$ authored several published novellas in Arabic, won a national poetry award and was the arts reviewer for the university 